\title{
Weak Localization in Ferromagnetic (Ga,Mn)As Nanostructures
}

\author{
D. Neumaier, ${ }^{*}$ K. Wagner, S. Geißler, U. Wurstbauer, J. Sadowski, W. Wegscheider, and D. Weiss \\ Institut für Experimentelle und Angewandte Physik, Universität Regensburg, 93040 Regensburg, Germany
} (Received 2 March 2007; published 13 September 2007)

\begin{abstract}
We report on the observation of weak localization in arrays of $(\mathrm{Ga}, \mathrm{Mn})$ As nanowires at millikelvin temperatures. The corresponding phase coherence length $L_{\phi}$ is typically between 100 and $200 \mathrm{~nm}$ at $20 \mathrm{mK}$. Strong spin-orbit interaction in the material is manifested by a weak antilocalization correction around zero magnetic field.
\end{abstract}

PACS numbers: 73.43.Jn, 72.25.Dc, 73.43.Qt

Quantum corrections to the resistance like weak localization are suppressed by a sufficiently strong perpendicular magnetic field $B$ [1]. Hence, the question arises whether such effects can be observed in ferromagnets having an intrinsic magnetic induction. While few experimental works explored this problem [2,3], a definite experimental answer is still lacking. Hence, the advent of the new ferromagnetic semiconductor (Ga,Mn)As with significantly smaller internal field compared to conventional ferromagnets offers a new opportunity to address such questions. The spin $\frac{5}{2}$-Mn-ions on regular sites of the zinc-blende lattice of the GaAs host act as acceptors, thus providing both holes and magnetic moments. The ferromagnetic order between the Mn-ions is mediated by these holes [4]. By now, ferromagnetism in $(\mathrm{Ga}, \mathrm{Mn}) \mathrm{As}$ is well understood, allowing to predict Curie temperatures [4], magnetocrystalline anisotropies, as well as the anisotropic magnetoresistance effect [6]. In this respect, $(\mathrm{Ga}, \mathrm{Mn}) \mathrm{As}$ is one of the best understood ferromagnetic materials at all [7] and hence suitable as a model system to study quantum corrections to the conductivity.

Interference effects originating from the charge carriers' wave nature are barely explored and understood in ferromagnets in general and in $(\mathrm{Ga}, \mathrm{Mn}) \mathrm{As}$ in particular. To this class of effects belong universal conductance fluctuations (UCF) [8], the Aharonov-Bohm (AB) effect [9], weak localization (WL) [1], weak antilocalization (WAL) [1], and electron-electron interactions (EEI) [10]. In (Ga,Mn)As, the phase coherence length was extracted from UCFs in nanowires giving typical values between 90 and $300 \mathrm{~nm}$ at $20 \mathrm{mK}$ [11,12]. This raises the question whether WL corrections - or WAL effects - can be observed in ferromagnetic $(\mathrm{Ga}, \mathrm{Mn}) \mathrm{As}$, a material in which the spin-orbit (SO) interactions for holes in the valence band is quite strong.

Below, we report the observation of WL and WAL in ferromagnetic $(\mathrm{Ga}, \mathrm{Mn}) \mathrm{As}$ wires and films, thus demonstrating that WL is not destroyed by the ferromagnets' magnetization. The effect of WL in disordered electronic systems - investigated intensively in the past for nonferromagnetic materials [13] — is due to quantum interference on time-reversed paths. This leads to an enhanced probability of backscattering. As an applied perpendicular
$B$-field suppresses the WL, the magnetoconductance is positive [1]. In the presence of SO interaction, the spin part of the wave function needs to be taken into account. The two partial waves on time-reversed closed paths experience a spin rotation in opposite direction causing (partially) destructive interference [1]. So, SO interactions leads to reduced backscattering and reverses the sign of the WL, hence called weak antilocalization. A typical signature of WAL is a double dip in the magnetoconductance trace [1].

For the experiments, two wafers having a $42 \mathrm{~nm}$ and a $20 \mathrm{~nm}$ thick $(\mathrm{Ga}, \mathrm{Mn})$ As layer were used. Both were grown by low-temperature molecular beam epitaxy deposited on semi-insulating $\mathrm{GaAs}(001)$ [14]. The nominal Mn concentration of the $42 \mathrm{~nm}$ layer was $5.5 \%$, of the $20 \mathrm{~nm}$ layer $5 \%$. The Curie temperature $T_{C}$ of the as grown layer was $90 \mathrm{~K}$ $(42 \mathrm{~nm})$ and $55 \mathrm{~K}(20 \mathrm{~nm})$, respectively. The samples' remanent magnetization was always in-plane. Some of the samples were annealed at $200{ }^{\circ} \mathrm{C}$ increasing both carrier density and $T_{C}$ [15]. To investigate phase coherent properties Hall-bar mesas, individual nanowires and arrays of wires were fabricated employing optical and electron beam lithography. For nanowire fabrication, we used a scanning electron microscope equipped with a nanonic pattern generator and subsequent reactive ion etching. Au contacts to the devices were made by lift-off technique. The characteristic parameters of the samples investigated are listed in Table I.

TABLE I. Length $L$, width $w$, and thickness $t$ of the samples. Some of the samples were annealed at $200{ }^{\circ} \mathrm{C}$. Resistivity $\rho$ and carrier concentration $n$ were taken at $T=300 \mathrm{mK}$.

\begin{tabular}{lrcccc}
\hline \hline \multicolumn{1}{c}{ Sample } & $1 \mathrm{a}$ & 2 & $2 \mathrm{a}$ & 3 & 4 \\
\hline$L(\mu \mathrm{m})$ & 60 & 7.5 & 7.5 & 7.5 & 0.37 \\
$w(\mathrm{~nm})$ & 7200 & 42 & 42 & 35 & 35 \\
$t(\mathrm{~nm})$ & 20 & 42 & 42 & 42 & 42 \\
Number of wires $N$ & 1 & 25 & 25 & 12 & 1 \\
$t_{\text {anneal }}$ at $200^{\circ} \mathrm{C}(\mathrm{h})$ & 8.5 & $\ldots$ & 51 & $\cdots$ & $\cdots$ \\
$n\left(10^{26} / \mathrm{m}^{3}\right)$ & 1.7 & 3.8 & 9.3 & 3.8 & 3.8 \\
$\rho\left(10^{-5} \Omega \mathrm{m}\right)$ & 13 & 3.5 & 1.8 & 3.5 & 3.5 \\
$T_{C}(\mathrm{~K})$ & 95 & 90 & 150 & 90 & 90 \\
\hline \hline
\end{tabular}


Magnetotransport was measured in a dilution refrigerator. To avoid heating, we used a low frequency $(19 \mathrm{~Hz})$ and low current (25 to $200 \mathrm{pA}$ ) four probe lock-in technique. As we see no effects of saturation for the different experiments (UCF, WL, and conductivity decrease) at low $T$, we assume that the effective electron temperature is in equilibrium with lattice and bath temperature even at $20 \mathrm{mK}$.

To search for WL effects in $(\mathrm{Ga}, \mathrm{Mn}) \mathrm{As}$ wires, we measured the resistance of $N$ parallel wires to suppress UCFs by ensemble averaging. A corresponding micrograph of sample 2 with 25 wires is shown in Fig. 1(a). The sample's conductance as a function of a perpendicular $B$ field is shown in Fig. 1(b) [16]. First, we start with a description of the dominant features observed in experiment. The pro-

a)
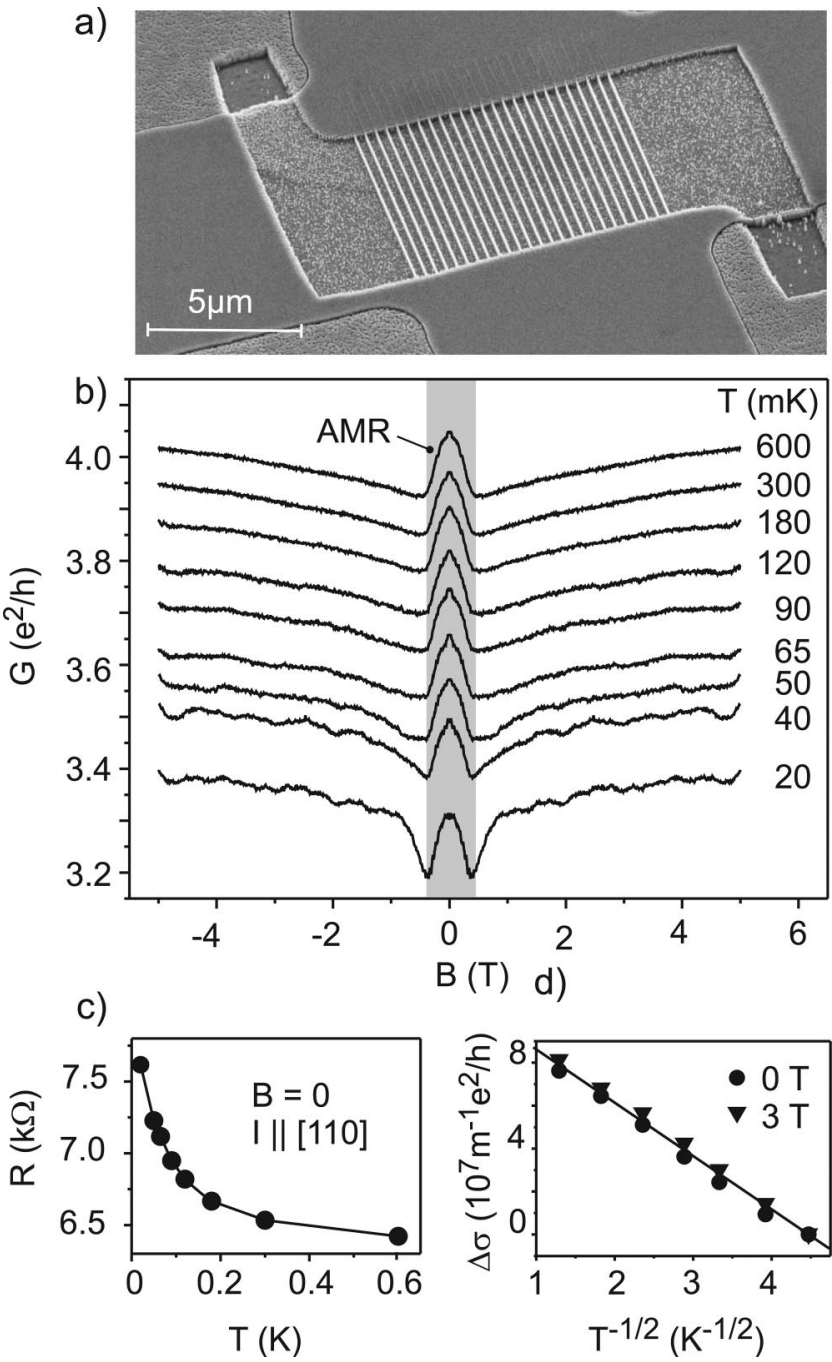

$\mathrm{T}(\mathrm{mK})$ 600 300 180 180 120
200 65 40 20

FIG. 1. (a) Electron micrograph of sample 2. (b) Conductance of sample 2 for different temperatures measured in a perpendicular magnetic field [29]. The magnetic field range where the magnetization is rotated from in-plane to out-of-plane is grayshaded. (c) Increase of resistance with decreasing temperature. This increase stems from EEI as proven by the $-T^{-1 / 2}$ power law for $1 \mathrm{D}$-systems at $B=0$ and $B=3 \mathrm{~T}$ in (d). Here, $\Delta G$ is taken relative to the conductivity at $50 \mathrm{mK}$. nounced conductance maxima around $B \sim 0$ are due to the anisotropic magnetoresistance (AMR) effect [6]. For an inplane magnetization, the conductance is higher than for an out-of-plane orientation of $M$ [17]. The positive slope of $G$ for higher $B$ is ascribed to increasing magnetic order [18] or to weak localization in bulk (Ga,Mn)As [19]. For temperatures larger than $\sim 65 \mathrm{mK}$, the different $G(B)$ traces are shifted but without noticeable change of shape, and the AMR peak height scales with the high-field background conductance. The decreasing $G$ for decreasing $T$ in Fig. 1(b) stems from the usual low $T$ behavior of the resistance in (Ga,Mn)As which is plotted in Fig. 1(c). With decreasing $T$, the resistance rises and is ascribed to EEI. Similar low $T$ behavior has been reported previously for conventional ferromagnets, too [3,20]. According to theory [21], the EEI conductivity correction for 1D systems goes with $-T^{-1 / 2}$. The corresponding conductance correction $\Delta \sigma=\sigma(T)-\sigma(50 \mathrm{mK})$ of our sample 2, taken at $B=0$ and at $B=3 \mathrm{~T}$, is plotted in Fig. 1(d) vs $T^{-1 / 2}$. The resulting straight lines for both $B$ values demonstrate the expected $T$ dependence, prove that the correction is independent of $B$, and hence suggest that EEI is accountable for the conductance decrease at low $T$. The novel features which are in the focus of this Letter appear at still lower temperatures. At about $50 \mathrm{mK}$, two downward cusps at $\sim \pm 0.4 \mathrm{~T}$ start to become noticeable and have developed to a prominent feature at $20 \mathrm{mK}$.

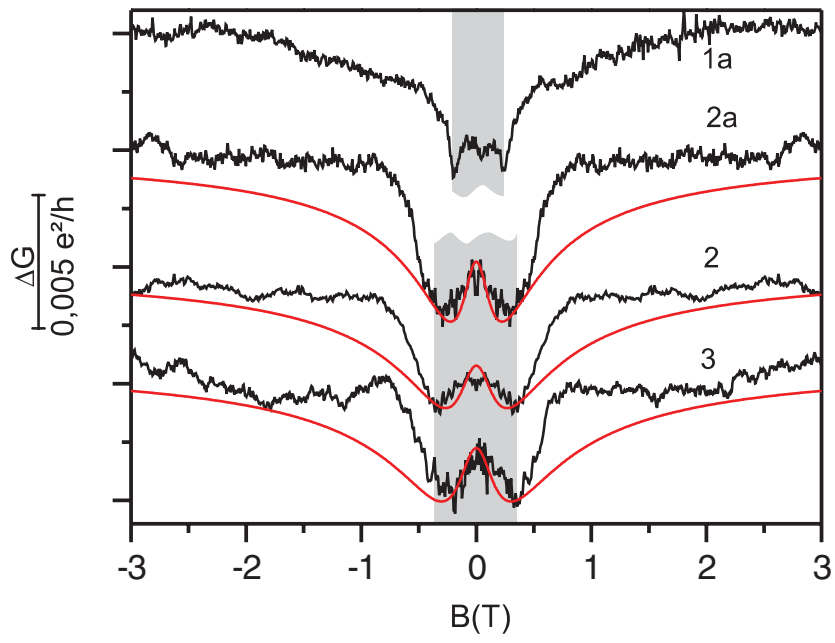

FIG. 2 (color). WL contribution for three wire and one 2D sample obtained after subtracting the $120 \mathrm{mK}$ trace as background conductance. To compare the different samples, the total $\Delta G$ was divided by the number of parallel wires. In case of the 2D-sample, 1a $\Delta G$ was divided by 15 to fit into the graph. Again, the gray shaded $B$-range corresponds to the regime where the samples's magnetization follows the external field and changes direction. The red lines are best fits to Eq. (1), discussed in the text. The fit parameters were $L_{\phi}=190 \mathrm{~nm}$ and $L_{\text {SO }}=113 \mathrm{~nm}$ for sample $2 \mathrm{a}, L_{\phi}=150 \mathrm{~nm}$ and $L_{\mathrm{SO}}=93 \mathrm{~nm}$ for sample 2, and $L_{\phi}=160 \mathrm{~nm}$ and $L_{\mathrm{SO}}=93 \mathrm{~nm}$ for sample 3 . Fitting the 2D sample requires a different formalism which is beyond the scope of the present Letter. 
To separate the peculiar low $T$ conductance features from the "high temperature" background, $\Delta G=$ $G(20 \mathrm{mK})-\alpha G(120 \mathrm{mK})$ of four samples was taken and plotted in Fig. 2. The factor $\alpha$ takes the $T$ dependence of $G$ into account and is given by $\alpha=$ $G(20 \mathrm{mK}) / G(120 \mathrm{mK})$, taken at $3 \mathrm{~T}$. We note, though, that putting $\alpha=1$ does not change $\Delta G$ qualitatively as the conductance change is only $\sim 10 \%$. To compare the different samples, $\Delta G$ was normalized by the number of parallel wires, $N$. All traces in Fig. 2 show a characteristic broad conductance minimum for $|B|<1 \mathrm{~T}$ and a local maximum at $B \sim 0 \mathrm{~T}$. Such $\Delta G(B)$ line shapes are characteristic for WAL in systems with spin-orbit interaction. Here, we have assumed that the $T$-dependency of the AMR contribution does not change for $T<65 \mathrm{mK}$.

To extract the characteristic lengths from the WL correction, we compare the data of Fig. 2 with existing theory. In Fig. 3(a), we particularly compare the WL correction of sample 3, with the standard expression for WL correction in 1D. Since the width $w$ and thickness $t$ of our wires are smaller than the phase coherence length $L_{\phi}$, which is expected to be of order $100 \mathrm{~nm}$ at $\sim 20 \mathrm{mK}$ [11], $w \sim t<$ $L_{\phi} \ll L$ holds and the 1D assumption is justified. The corresponding equation for the conductance correction reads $[22,23]$

$$
\begin{aligned}
\Delta G= & g_{s} \frac{e^{2}}{h}\left[\frac{1}{2 L}\left(\frac{1}{L_{\phi}^{2}}+\frac{1}{3} \frac{w^{2}}{L_{H}^{4}}\right)^{-1 / 2}\right. \\
& \left.-\frac{3}{2 L}\left(\frac{1}{L_{\phi}^{2}}+\frac{4}{3 L_{S O}^{2}}+\frac{1}{3} \frac{w^{2}}{L_{H}^{4}}\right)^{-1 / 2}\right],
\end{aligned}
$$

where $g_{s}$ is the spin degeneracy. Here, $L_{\mathrm{SO}}=\sqrt{D \tau_{\mathrm{SO}}}$ is the spin-orbit length that characterizes the strength of spinorbit coupling, $L_{\phi}=\sqrt{D \tau_{\phi}}$, and $L_{H}=\sqrt{\hbar / e B}$ is the magnetic length. Equation (1) is fitted to the WL data in Fig. 3(a) for sample 3. As the valence band is spin split, the holes are highly (but not fully) spin polarized [24]. To account for spin polarization, we approximate $g_{s}$ either by 1 (fully spin polarized) or by 2 (spin degenerate) as adjustable parameter. While the fit for $g_{s}=1$ matches the conductance minima at $\pm 400 \mathrm{mT}$ as well as the conductance correction $\Delta G$, the fit for $g_{s}=2$ is less satisfying. The parameters used for the fit were $L_{\phi}=160 \mathrm{~nm}, L_{\mathrm{SO}}=$ $93 \mathrm{~nm}$ for $g_{s}=1$ and $L_{\phi}=90 \mathrm{~nm}, L_{\mathrm{SO}}=50 \mathrm{~nm}$ for $g_{s}=2$, respectively. Also, the WL data of the other samples can be modeled by Eq. (1) and $g_{s}=1$; the corresponding fits and parameters are given in Fig. 2.

The size of the weak (anti)-localization contribution in Fig. 2 and 3(a) is quite nicely fitted by two parameters, $L_{\phi}$ and $L_{\mathrm{SO}} . L_{\phi}$ can also be extracted from UCFs measured on individual 1D-wires $[11,12]$. To study UCFs, we fabricated a single wire, $w=35 \mathrm{~nm}$ wide and $L=370 \mathrm{~nm}$ long, from the same material as sample 2 and 3 (sample 4 in Table I). A corresponding electron micrograph is shown as lower left inset in Fig. 3(b). $G(B)$ was measured in a perpendicular $B$-field from $-3 \mathrm{~T}$ to $3 \mathrm{~T}$ for $T$ between

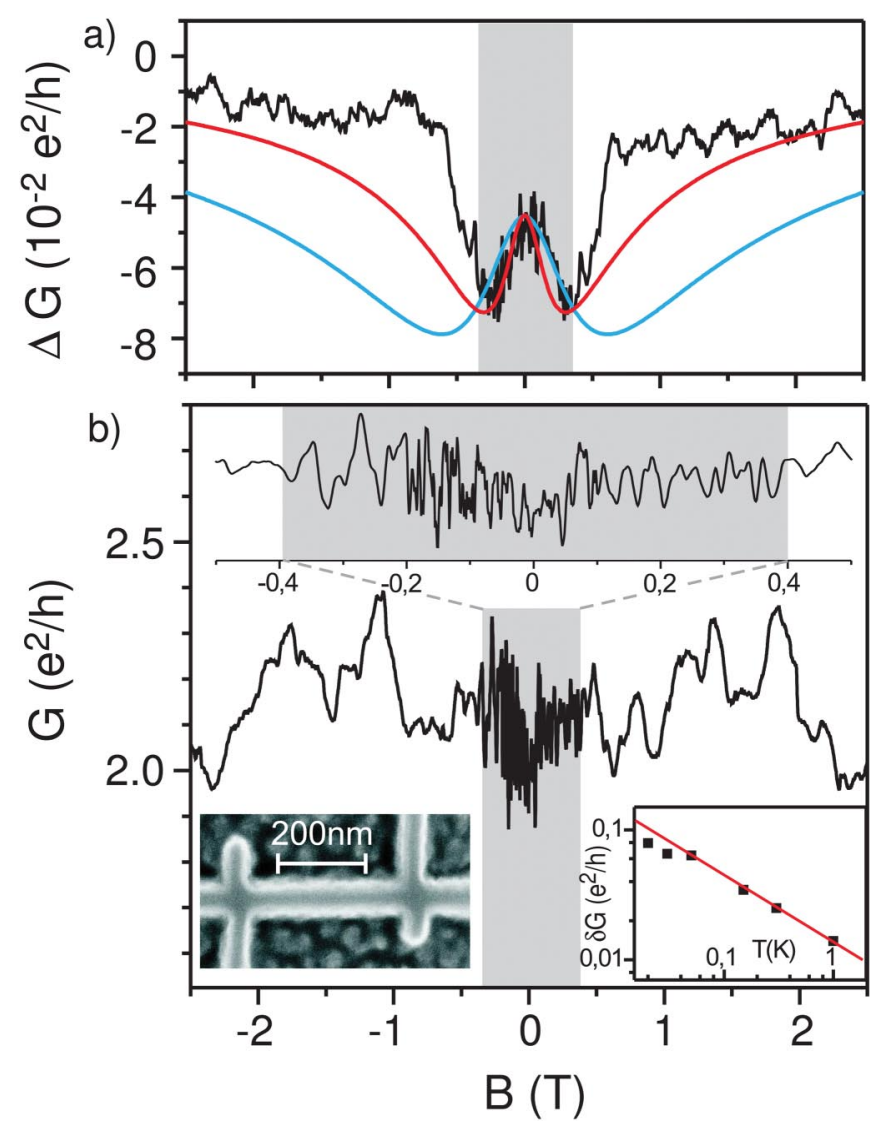

FIG. 3 (color). (a) WL correction of sample 3 fitted with the standard 1D WL theory [Eq. (1)] for spin degeneracy $g_{s}=1$ (red) and $g_{s}=2$ (blue). (b) UCFs measured in an individual 1Dwire made from the same material (sample 4). An electron micrograph of the wire is shown in the lower left inset. The gray shaded regime again corresponds to the magnetic field range where $M$ changes direction. The upper inset shows the low-field UCFs in a expanded magnetic field scale. The temperature dependence of $\delta G$, extracted from the low-field fluctuations, is shown in the lower right inset.

$20 \mathrm{mK}$ and $1 \mathrm{~K}$ (for details see [11]). Corresponding data taken at $20 \mathrm{mK}$ show pronounced, reproducible UCFs, displayed in Fig. 3(b). The root mean square amplitude $\delta G_{\mathrm{rms}}=\sqrt{\left\langle(G-\langle G\rangle)^{2}\right\rangle}$ of these fluctuations is connected with $L_{\phi}$, and the wire length $L$ by $\delta G_{\text {rms }} \approx\left(e^{2} / h\right) \times$ $\left(L_{\phi} / L\right)^{3 / 2} f\left(L_{\phi} / L_{\mathrm{SO}}\right)$ [25]. The function $f\left(L_{\phi} / L_{\mathrm{SO}}\right)$ takes spin-orbit interaction into account. For $L_{\phi} / L_{\mathrm{SO}} \sim 1.7$, we obtain $f\left(L_{\phi} / L_{\mathrm{SO}}\right) \sim 0.56$ [25]. Extracting $L_{\phi}$ from $\delta G_{\mathrm{rms}}$, taking only the fluctuations between $\pm 400 \mathrm{mT}$ in Fig. 3(b) into account, results then in $L_{\phi} \sim 118 \mathrm{~nm}$. The value of $L_{\phi}$, extracted from UCFs, is thus in surprisingly good agreement with the ones used to fit the WL correction. Hence, our analysis suggests that $L_{\mathrm{SO}}$ ranges between $\sim 93 \mathrm{~nm}$ and $\sim 113 \mathrm{~nm}$ in our devices.

While WAL was observed, e.g., in nonmagnetic $p$-type $(\mathrm{Al}, \mathrm{Ga}) \mathrm{As} / \mathrm{GaAs}$ quantum wells [26] or in (In,Ga)As quantum wells [27], the observation of WAL-signature in ferromagnetic $(\mathrm{Ga}, \mathrm{Mn}) \mathrm{As}$ comes as a surprise. A recent 
theory suggests the processes, leading to WAL in nonmagnetic systems, to be totally suppressed in ferromagnets [28]. The suppression of WAL in ferromagnets is due to the strong magnetic polarization which excludes contributions from the so-called singlet Cooperon diagrams, responsible for WAL. As a consequence, the quantum correction to $G$ is expected to be exclusively negative in ferromagnets, leading to positive magnetoconductance. This clearly contradicts our experimental observation.

While the fits in Figs. 2 and 3(a) are in good agreement with experiment for $|B|<400 \mathrm{mT}$, the concordance at larger $B$ is less perfect. The WL or WAL correction is, as a function of increasing $B$, more abruptly suppressed than expected from theory. There is a striking correlation with the magnetic field dependence of the AMR effect. The magnetic field region where the AMR occurs is highlighted by gray shading in Figs. 1(b), 2, 3(a), and 3(b). Within this $B$-field range, the magnetization is rotated from in-plane to out-of-plane. Once the magnetization is out-of-plane, the WL correction drops quickly. At the same magnetic field, the magnetic length matches wire width and thickness, $L_{H} \sim w, t$. Hence, the discrepancy between fit and experiment might be associated with dimensional crossover (1D to $3 \mathrm{D}$ ), if $|B|$ exceeds $400 \mathrm{mT}$ and Eq. (1) might be inapplicable. In the gray shaded $B$-field range, the fluctuations of an individual wire show a reduced correlation field $B_{C}$. Corresponding data are displayed in Fig. 3(b), magnified in the upper inset. Similar behavior was observed in previous experiments on samples with in-plane easy axis $[11,12]$ and ad hoc ascribed to the formation of domain walls in [12]. Though we can not exclude such a scenario, we note that $B_{C}$ is not a well defined quantity in the regime where the (magnetic) configuration changes.

The observation of WAL, contrary to theoretical expectation, the abrupt suppression of the WL correction once the magnetization is saturated, as well as the anomalous $B_{C}$ in the low $B$-regime suggest that some important ingredients are still missing to describe interference phenomena in $(\mathrm{Ga}, \mathrm{Mn})$ As. This is not too surprising as neither the field dependent change of the magnetization direction nor the $\frac{3}{2}$-spin of the involved hole states was taken into account. Especially, the latter could add a number of additional interference diagrams not yet treated theoretically.

In summary, we have shown that quantum inference effects strongly affect the low-temperature conductance of ferromagnetic (Ga,Mn)As. By resolving a clear weak localization signature, we demonstrate that interference due to scattering on time-reversed paths can exist also in ferromagnetic materials with internal magnetic induction. The strong spin-orbit interaction in $(\mathrm{Ga}, \mathrm{Mn}) \mathrm{As}$ is manifested by a weak antilocalization contribution at low $B$.
We thank K. Richter, I. Adagideli, J. Fabian, T. Dietl, A. Geim, and J. Nitta for valuable discussions. Financial support by the Deutsche Forschungsgemeinschaft (DFG) via SFB 689 is gratefully acknowledged.

*daniel.neumaier@physik.uni-regensburg.de

[1] G. Bergmann, Phys. Rep. 107, 1 (1984).

[2] M. Aprili et al., Solid State Commun. 102, 41 (1997).

[3] M. Brands et al., Phys. Rev. B 72, 085457 (2005).

[4] T. Dietl et al., Science 287, 1019 (2000).

[5] M. Sawicki, J. Magn. Magn. Mater. 300, 1 (2006) and references therein.

[6] D. V. Baxter et al., Phys. Rev. B 65, 212407 (2002).

[7] T. Jungwirth et al., Rev. Mod. Phys. 78, 809 (2006).

[8] P. A. Lee, A. D. Stone, and H. Fukuyama, Phys. Rev. B 35, 1039 (1987).

[9] S. Washburn and R. Webb, Adv. Phys. 35, 375 (1986).

[10] B. L. Altshuler, A. G. Aronov, and P. A. Lee, Phys. Rev. Lett. 44, 1288 (1980).

[11] K. Wagner et al., Phys. Rev. Lett. 97, 056803 (2006).

[12] L. Vila et al., Phys. Rev. Lett. 98, 027204 (2007).

[13] J. J. Lin and J. P. Bird, J. Phys. Condens. Matter 14, R501 (2002).

[14] M. Reinwald et al., J. Cryst. Growth 278, 690 (2005).

[15] K. W. Edmonds et al., Phys. Rev. Lett. 92, 037201 (2004).

[16] In ferromagnetic wires the total magnetic field $B_{z}^{\prime}$ in z-direction is given by $B_{z}^{\prime}=B_{z}+J_{z} / 2$ with the external field $B_{z}$, the magnetic polarization $J_{z}$, and the demagnetization factor in $z$-direction, $\sim 1 / 2$. As the maximum (saturation) value of $J_{z}$ in our samples is only of order $40 \mathrm{mT}$, we approximate $B_{z}^{\prime} \approx B_{z}=B$.

[17] H. Ohno, Science 281, 951 (1998).

[18] E. L. Nagaev, Phys. Rev. B 58, 816 (1998).

[19] F. Matsukura et al., Physica E (Amsterdam) 21, 1032 (2004).

[20] T. Ono et al., J. Magn. Magn. Mater. 226, 1831 (2001).

[21] P. A. Lee and T. V. Ramakrishnan, Rev. Mod. Phys. 57, 287 (1985).

[22] B. L. Altshuler and A. G. Aronov, JETP Lett. 33, 499 (1981).

[23] F. Pierre et al., Phys. Rev. B 68, 085413 (2003).

[24] J. G. Braden et al., Phys. Rev. Lett. 91, 056602 (2003).

[25] V. Chandrasekhar, P. Santhanam, and D. E. Prober, Phys. Rev. B 42, 6823 (1990).

[26] S. Pedersen et al., Phys. Rev. B 60, 4880 (1999).

[27] T. Koga et al., Phys. Rev. Lett. 89, 046801 (2002).

[28] V. K. Dugaev, P. Bruno, and J. Barnaś, Phys. Rev. B 64, 144423 (2001).

[29] In sample 2 and $2 \mathrm{a}$, the two voltage leads were fabricated on opposite sites. So, also the Hall conductance was measured. To remove the Hall conductance from the data, only the symmetric part of the trace was taken. As this leads to the same result then seen on sample 1a and 3, where the voltage leads are on the same side, physics is not changed by this procedure. 